\title{
Characterization of Fine Metallic Soap Particles by X-Ray Diffraction, Differential Scanning Calorimetry, and Specific Surface Area Analysis
}

\author{
Kouhei SAWADA ${ }^{1 *}$ and Miki KonAKA ${ }^{1}$ \\ ${ }^{I}$ Oleochemicals Research Laboratory, NOF Corporation \\ (Amagasaki, Hyogo 660-0095, JAPAN)
}

Edited by Y. Takizawa, Tokyo Gakugei Univ., and accepted July 1, 2004 (received for review June 3, 2004)

\begin{abstract}
Fine metallic soap particles synthesized by jet mixing process have a much narrower particle size distribution and more homogeneous surface structure than metallic soap particles prepared by the method of pulverizing and classifying. With jet mixing, reaction conditions can be adjusted so as to control particle sizes while maintaining the above characteristics unchanged. With these particles it is possible to examine in detail the relationship between the particle size and condition and the crystalline condition and other physical properties, which has been considered impossible to perform using metallic soaps prepared by the conventional method of pulverizing and classifying. So, using fine particles of fatty acid zinc salt as a model of metallic soap for structure analysis, the influence of average particle size and fatty acid composition were examined on crystallinity, heat characteristics and other properties of the particles. Diffraction intensities, half-band widths, and long spacings of Miller index (003) peaks were analyzed from X-Ray Diffraction chart and compared with average particle size and fatty acid composition for fine particles of fatty acid zinc salt. Furthermore, Crystallinity of fine particles of fatty acid zinc salt were examined by these results, and were compared with results of differential scanning calorimetry and specific surface area analysis. The results are described in the present report.
\end{abstract}

Key words: fine metallic soap particles, X-ray diffraction, crystal structure, specific surface area, differential scanning calorimetry

\section{Introduction}

In the cutting-edge industries, such as the electronics information industry, there have been rising demands in recent years for the development of fine-particle materials that enable higher functionality. Improvement of functions and development of new functions by the particle miniaturization are the subjects of particular attention (1). For example, carbon nanotubes, fullurenes, and nano-metal oxides, which are ultra-fine particles prepared from carbon and other well-known inorganic mat- ters, provide innovative electric and heat characteristics and other application properties (2). Though made of common materials, these fine particles with characteristic structures and shapes are expected to exert functions that make them novel functional materials.

Fatty acids and synthetic materials made from fatty acids (hereafter "fatty acid derivatives") have been contributing greatly to the development of industries worldwide and are still in use in a broad variety of areas. The most common fatty acid derivatives are multivalent metal salts of long-chain fatty acids (hereafter "metallic

\footnotetext{
*Correspondence to: Kouhei SAWADA, Oleochemicals Research Laboratory, NOF Corporation, 1-56 Oohama-cho, Amagasaki-shi, Hyogo 6600095, JAPAN

E-mail: kouhei_sawada@nof.co.jp
} 


\section{K. Sawada and M. Konaka}

soaps"). When added to resins and oil solutions, they fulfill diverse functions, such as high lubricating property, water repellency and electrification property (3). Metallic soaps are obtained mainly in the form of powder, and the particle shape has influence on the diverse functions mentioned above. This means that if it becomes possible to produce fine metallic soap particles with characteristic shapes, metal soaps will be transformed into new functional materials with innovative functions.

We started to work on the particle miniaturization of fine metallic soap particles in 1996, and in 2000 we brought to completion the world's first practical production method (jet-mixing) of fine metallic soap particles that does not require pulverizing (4). Using this production method it is now possible to synthesize fine metallic soap particles with average diameters ranging from $0.3 \mu \mathrm{m}$ to $3 \mu \mathrm{m}$. These particles possess various properties and applications that common metallic soaps did not have, and their usefulness in the fields of electronics information, high-precision processing and many other cutting-edge industries has been confirmed (5-7).

Furthermore, as pulverizing is not necessary in the synthesis of metallic soap powder by the jet-mixing method, no mechanical fracture energy is applied on the particles, so that no fracture surfaces are created. Therefore, it is now possible, while keeping the conditions of particle formation almost identical, to produce particles with any arbitrary average diameter between $0.3 \mu \mathrm{m}$ and $3 \mu \mathrm{m}$ without changing particle shapes, which had been difficult to achieve using the pulverization method. To date, there has been a lot of research on how the kind of central metal in the metallic soap and chain length influence the crystal structure and physical properties (8-11). However, for the metallic soap particles prepared by conventional method of pulverizing and classifying, it has not been possible to evaluate physical properties and structures under conditions where the formation conditions of particles and their sizes were held identical. For the fine metallic soap particles synthesized by jet mixing, because of their above mentioned characteristics, it is possible to study in detail the relationship between particle size, particle state, crystalline state, and other physical property values. Using fine particles of fatty acid zinc salt as a model for structure analysis of metallic soap, we examined the influence of average particle size and fatty acid composition on crystallinity, heat characteristics and other properties of the particles. The results are described in the present report.

\section{Experimental}

\section{$\mathbf{2} \cdot \mathbf{1}$ Material}

We synthesized zinc myristate (C14FA-Zn), zinc palmitate (C16FA-Zn), zinc stearate (C18FA-Zn), and zinc salts prepared from a fatty acid mixture of stearic and palmitic acids (C16/C18FA-Zn) as fine metallic soap particles. For the synthesis, stearic acid of $>98 \%$ purity (NAA180, NOF Corporation), palmitic acid of $>98 \%$ purity (NAA160, NOF Corporation), and zinc sulfate of $>97 \%$ purity (Katayama Chemical, Inc.) were used. Purified water was used for the reactions and rinsing.

Figure 1 shows the equipment used for the jet-mixing synthesis of fine particles of fatty acid zinc salt (4). An aqueous solution of alkaline salt of fatty acid kept at $60^{\circ} \mathrm{C}-80^{\circ} \mathrm{C}$ and an aqueous solution of zinc sulfate (at an equivalent ratio of about 1:1.1) were injected continuously under pressure at the same weights into a narrow void for jet mixing, followed by quick discharge from the reaction system. Thus a suspension of fine particles of fatty acid zinc salt was obtained. The suspension was cooled rapidly, filtered, rinsed with water and dried to give fine particles of fatty acid zinc salt as a dry powder. "Zinc stearate" produced by NOF, Co. Ltd. (average diameter $4.9 \mu \mathrm{m}$ ) and its pulverized product (average diameter $3.5 \mu \mathrm{m}$ ) was used as sample of common zinc stearate for comparison with the above fine metallic soap particles. Zinc stearate is a fatty acid zinc salt produced from a mixture of stearic acid and palmitic acid as raw materials. The ratio of stearic acid to palmitic acid is $70: 30$ by weight.

\section{2·2 Scanning Electron Micrograph Imaging}

A sample of fine particles of fatty acid zinc salt was attached to an aluminum plate using double-faced adhesive tape, coated by gold evaporation, and observed with a scanning electron microscope (2100A, Hitachi, Ltd.).

\subsection{Particle Size Distribution Measurement}

In a $100 \mathrm{ml}$ beaker, $0.01 \mathrm{~g}$ of fine particles of fatty acid zinc salt, $0.1 \mathrm{~g}$ of dispersing agent, and $40 \mathrm{ml}$ of 


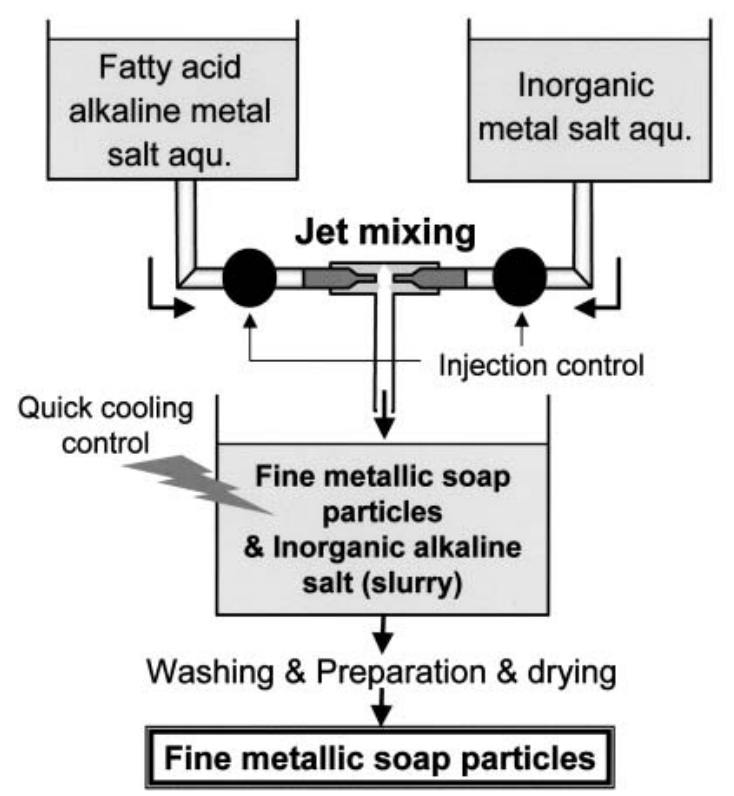

Fig. 1 The Image Showing new Producing Process of the Fine Metallic Soap Particles (Jet-Mixing process).

purified water were added and homogenized at 4000 rpm for 3 minutes with a homogenizer (US-50, Nihon Seiki). The average particle size, particle size distribution, and standard deviation of the suspension obtained were measured using a laser diffraction particle size analyzer (SALD-2000, Shimadzu Corporation). The standard deviation was obtained by substituting $\mu$ of the average particle size $\left(10^{\mu}\right)$, particle size $\mathrm{X}_{\mathrm{j}}(\mu \mathrm{m})$ and difference $q_{j}(\%)$ for distribution of the frequencies into the equation 1 (12).

Standard deviation $=$

$$
\sqrt{\left\{\frac{1}{100} \sum_{\mathrm{j}=1 \mathrm{q}_{\mathrm{j}+1}}^{\mathrm{n}-1}\left(\frac{\log _{10} \mathrm{x}_{\mathrm{j}}+\log _{10} \mathrm{x}_{\mathrm{j}+1}}{2}\right)\right\}^{2}-\mu^{2}}
$$

\subsection{X-Ray Diffraction Measurement}

Each sample of fine particles of fatty acid zinc salt was placed on a special glass sample plate for X-ray diffraction measurement with an X-ray powder diffractometer (vertical-axis, RINT4000, Rigaku Corporation). We focused our attention on the miller index (003) and determined its long spacing, diffraction intensity, and half-band width. The X-ray diffraction measurements were carried out using $\mathrm{CuK}_{\alpha}$ as a characteristic X-ray radiation source, at $40 \mathrm{mV}, 20 \mathrm{~mA}$, a scanning speed of $4^{\circ} / \mathrm{min}$, and diffraction angles of $5^{\circ}$ to $40^{\circ}$.

\subsection{Specific Surface Area Measurement}

The specific surface area of each sample of fine particles of fatty acid zinc salt was determined by gas sorption. In a special cell, $0.2 \mathrm{~g}$ of each sample was placed and the specific surface area was measured by fivepoint BET-analysis using an automatic specific surface area analyzer (GEMINI 2370, Shimadzu Corporation). Nitrogen was used as adsorption gas and helium was used as flow carrier gas.

\subsection{Differential Scanning Calorimetry}

Using $10 \mathrm{mg}$ of each sample of fine particles of fatty acid zinc salt, differential scanning calorimetry was measured with differential scanning calorimeter DSC6200 (Seiko Instruments Co.) at temperatures between $50^{\circ} \mathrm{C}$ and $200^{\circ} \mathrm{C}$ rising at a rate of $3^{\circ} \mathrm{C} / \mathrm{min}$.

\section{Results and Discussion}

\subsection{Shape and Size Distribution of Fine Particles of Fatty Acid Zinc Salt}

The electron microscope images $(5,000 \mathrm{X})$ of the common zinc stearate (average diameter: $4.9 \mu \mathrm{m}$ ) and C18FA-Zn (average diameter $0.8 \mu \mathrm{m}$ ) are shown in Fig. 2. The common zinc stearate is a heterogeneous aggregate of large crystals. Because the particles are produced by pulverization, there are many rough areas on the surface. C18FA-Zn particles, on the other hand, are minute and uniform in size with an average thickness of about $0.1 \mu \mathrm{m}$, appearing as lamellar-shaped particles with few rough areas. Formation of lamellar-shaped particles is observed not only with fine zinc stearate particles; this is a characteristic common to all other fatty acid salts of divalent metals synthesized by jet mixing (4).

It is a known fact that the shape of fine metallic soap particles depends on the kind of metal (4). For example, fine zinc stearate particles, fine calcium stearate particles and fine barium stearate particles are known as flake-shaped particles, whereas the fine magnesium stearate particles are strip-shaped, and the fine copper stearate particles are strip- or needle-shaped. The difference in the properties of particles depending on the kind of metal is thought to be attributable to the conjugation state of the metal with carboxyl group in fatty acid, but details have not been known to date. 


\section{K. Sawada and M. Konaka}

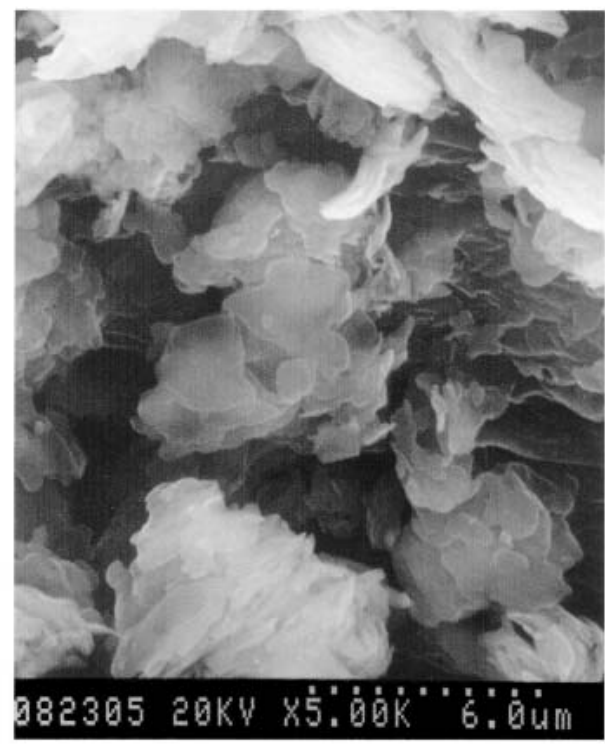

\section{Common zinc stearate}

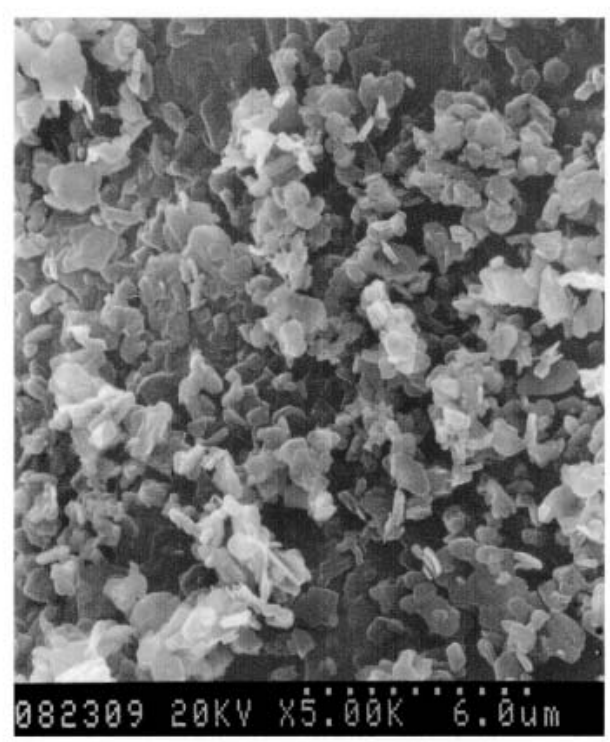

Fine zinc stearate (C18FA-Zn)

Fig. 2 Scanning Electron Micrograph Images for Common Zinc Stearate Particles and Fine Zinc Stearate Particles $(1.0 \mu \mathrm{m})$ at a Magnification of $5,000 \mathrm{X}$.
The size of fine particles of fatty acid zinc salt can be controlled arbitrarily by changing the reaction temperature or the retention conditions for the reaction suspension during jet mixing (4). The most minute fine metallic soap particle obtained so far has an average particle size of $0.3 \mu \mathrm{m}$ (7). By controlling the retention temperature of the reaction suspension, the average particle size can be increased up to $10 \mu \mathrm{m}$ at the maximum. Figures 3(1) to 3(3) show electron micrograph images of $\mathrm{C} 18 \mathrm{FA}-\mathrm{Zn}$ with an average particle size of $0.8,1.9$, or $3.1 \mu \mathrm{m}$ at a magnification of $10,000 \mathrm{X}$ (5). The images show that the flake-shaped C18FA-Zn particles are maintained when they increase in size. From the studies to date, the particles are found to have aspect ratios of 1.4 to 1.5 , meaning a relatively high circularity. This characteristic growth of particles is understood as a result of aggregation of flake-shaped particles through surface contact and concurrent translocation of molecules occurring within the aggregate according to the Ostwald ripening (4).

Next, particle size distribution of the common zinc stearate (average diameter $4.9 \mu \mathrm{m}$ ) and C18FA-Zn (average particle size $0.8 \mu \mathrm{m}$ ) are shown in Fig. 4. Furthermore, standard deviations of C14FA-Zn (average diameter $1.1 \mu \mathrm{m}$ ), C16FA-Zn (average particle sizes $0.8,1.1,1.4,1.8 \mu \mathrm{m}$ ), C18FA-Zn (average particle sizes 0.8, 1.3, 1.6, $1.9 \mu \mathrm{m}$ ), C16/C18FA-Zn (palmitic acid : stearic acid $=30 \mathrm{wt} \%: 70 \mathrm{wt} \%$, average particle sizes $0.7,1.0,1.3,1.9 \mu \mathrm{m})$, and of the common zinc stearate (average particle size $4.9 \mu \mathrm{m}$ ) are shown in Table 1 together with the particle sizes at the time when cumulative frequencies reached $90 \%$ (to be referred to as "particle size at 90\%"). Figure 1 show that the particles of C18FA-Zn synthesized by jet mixing have smaller sizes and that their size distribution is narrower as compared to the common zinc stearate. Table 1 show that the particle sizes at $90 \%$ of C14FA-Zn, C16FA-Zn, C18FA-Zn and C16/C18FA-Zn are all below $5 \mu \mathrm{m}$, which is smaller than the 2.5 -fold of the average diameter. This value is much less than the value observed with the common zinc stearate particles (6-fold). The standard deviations of C16FA-Zn, C18FA-Zn, $\mathrm{C} 16 / \mathrm{C} 18 \mathrm{FA}-\mathrm{Zn}$ are as small as 0.2 to 0.4 . From the above results, jet mixing, as compared to the common pulverizing method, is considered to be more suitable for obtaining particles of desired sizes while keeping the particle size distribution narrow. 


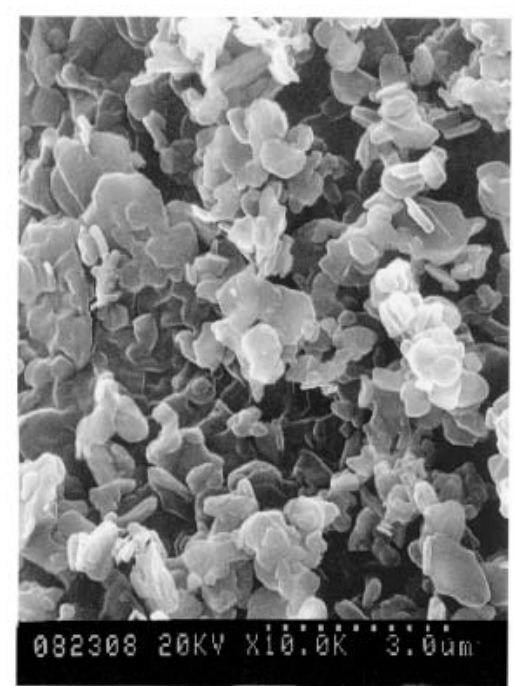

(1) $0.8 \mu \mathrm{m}$

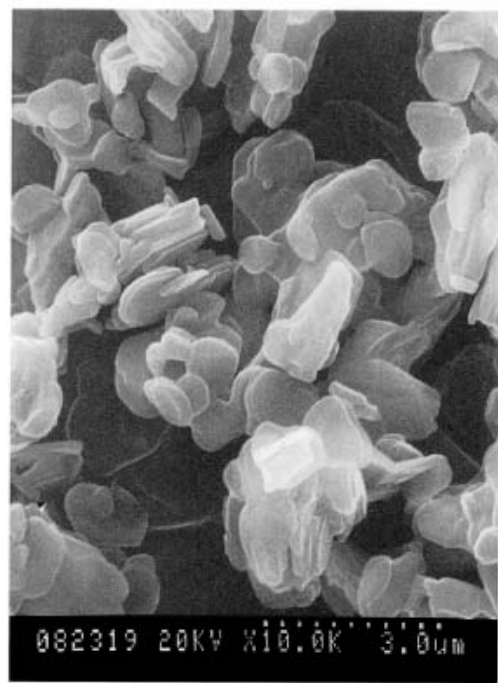

(2) $1.9 \mu \mathrm{m}$

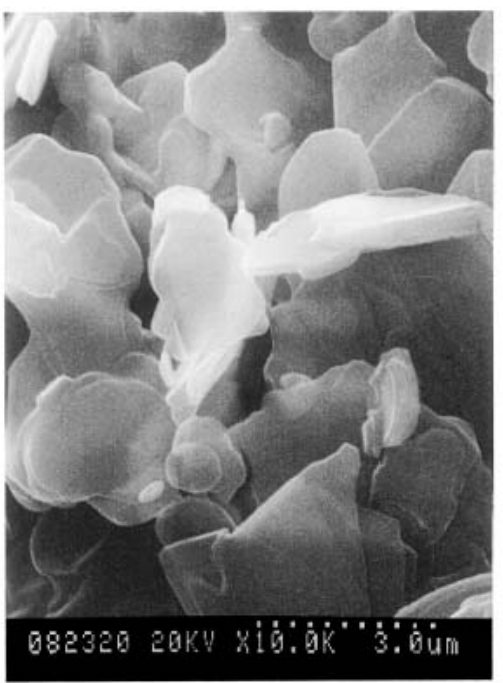

(3) $3.1 \mu \mathrm{m}$

Fig. 3 Scanning Electron Micrograph Images for Fine Metallic Soap Particles at a Magnification of 10,000X. Average particle sizes of fine metallic soap (1), (2), (3) are $0.8 \mu \mathrm{m}, 1.9 \mu \mathrm{m}, 3.1 \mu \mathrm{m}$ respectively.

\subsection{Relationship between Crystallinity and Particle Size of Fine Particles of Fatty Acid Zinc Salt}

As explained in the previous section, the fine metallic soap particles synthesized by jet mixing are uniform

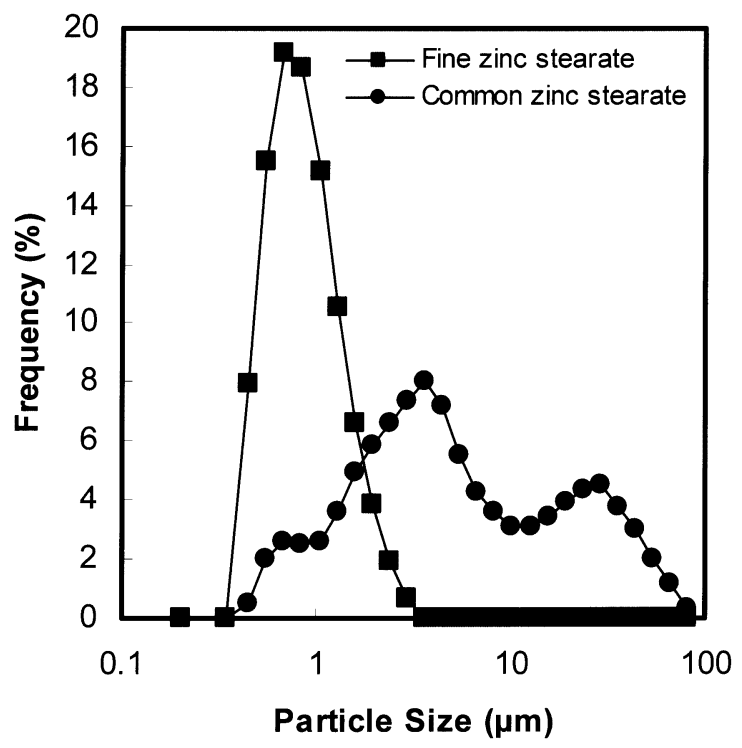

Fig. 4 Particle Size Distributions of Common Zinc Stearate Particles and Fine Zinc Stearate Particles (C18FA$\mathrm{Zn})$. These average particle sizes are $0.8 \mu \mathrm{m}$ and 4.9 $\mu \mathrm{m}$ respectively. and simple in shape, and the shape is maintained even if the particles grow larger. These facts suggest that the formation of metallic soap particles and crystal growth are controlled by relatively simple molecular arrays. Therefore, the fine metallic soap particles obtained by the new synthetic method are suitable for studying the influence of particle sizes on the crystal state and other properties of these particles, the analyses of which have been difficult so far.

Figure 5 show that the results of X-ray diffraction measurements of C18FA-Zn (average diameter $0.8 \mu \mathrm{m}$ ). Metallic soaps such as zinc stearate possess long fatty chains are considered to form one unit cell by aggregation of several molecules (8). Generally, the length of a unit cell represents a repetition unit, and the repeating interval is called the long spacing (10). In molecular crystals, the Miller index of long spacing is expressed as $(00 \mathrm{~L})$ and represents, for example, the distance from a metal to the next metal in a metallic soap crystal. If the largest spacing distance $\mathrm{d}$, which corresponds to the long spacing, is defined as $\mathrm{d}(001)$, and the L-th smallest spacing distance is defined as $\mathrm{d}(00 \mathrm{~L})$, then the relation $d(001)=d(00 L \times L$ exists for $d(00 L)(8)$. In the present study, we focused our attention on the diffraction peaks on the small-side angle in the X-ray diffraction chart. The spacing distance $d$ was calculated by substituting into Bragg's equation the $2 \theta$ values correspond- 


\section{K. Sawada and M. Konaka}

Table 1 Results of Particle Size Distribution Analysis, X-Ray Diffraction Analysis, Specific Surface Area Analysis, Density Analysis, Differential Scanning Calorimetry Analysis and Fitting Parameters of Specific Surface Area Simulation (x, y) for Fine Metallic Soap Particles (C14FA-Zn, C16FA-Zn, C18FA-Zn and C16/C18FA-Zn and Common Zinc Stearate Particles. Ratio of stearic acid in C16/C18FA-Zn is $70 \mathrm{wt} \%$.

\begin{tabular}{|c|c|c|c|c|c|c|c|c|c|c|c|c|}
\hline & $\begin{array}{c}\text { Average } \\
\text { particle } \\
\text { size } \\
(\mu \mathrm{m})\end{array}$ & $\begin{array}{l}\text { Standard } \\
\text { deviation }\end{array}$ & $\begin{array}{c}\text { Particle } \\
\text { size at } \\
90 \% * \\
(\mu \mathrm{m}))\end{array}$ & $\begin{array}{l}\text { Long } \\
\text { spacing }\end{array}$ & $\begin{array}{c}\text { Diffraction } \\
\text { intensity }\end{array}$ & $\begin{array}{l}\text { Half-band } \\
\text { width of } \\
\text { the } \\
\text { maximum } \\
\text { peak }\end{array}$ & $\begin{array}{c}\text { Specific } \\
\text { surface } \\
\text { area } \\
\left(\mathrm{m}^{2} / \mathrm{g}\right)\end{array}$ & $\begin{array}{c}\text { Density } \\
\rho \\
\left(\mathrm{g} / \mathrm{cm}^{3}\right)\end{array}$ & $\begin{array}{c}\text { Melting } \\
\text { point } \\
\left({ }^{\circ} \mathrm{C}\right)\end{array}$ & $\begin{array}{c}\Delta \mathrm{H} \\
(\mathrm{mJ} / \mathrm{mg})\end{array}$ & $\mathrm{x}$ & $\mathrm{y}$ \\
\hline $\begin{array}{l}\text { C14FA- } \\
\text { Zn }\end{array}$ & 1.1 & 0.20 & 2.1 & 33.9 & 1440 & 0.25 & 6.3 & 1.16 & 132.0 & 178.0 & & \\
\hline \multirow{4}{*}{$\begin{array}{c}\text { C16FA- } \\
\text { Zn }\end{array}$} & 0.8 & 0.18 & 1.4 & 38.3 & 945 & 0.23 & 9.5 & \multirow{4}{*}{1.14} & 132.4 & 183.2 & \multirow{4}{*}{4.5} & \multirow{4}{*}{2.3} \\
\hline & 1.1 & 0.20 & 1.9 & 38.3 & 1605 & 0.21 & 7.5 & & 131.7 & 187.9 & & \\
\hline & 1.4 & 0.23 & 2.5 & 38.2 & 1976 & 0.19 & 6.7 & & 131.9 & 189.5 & & \\
\hline & 1.8 & 0.25 & 3.9 & 38.3 & 2081 & 0.16 & 5.4 & & 132.0 & 190.6 & & \\
\hline \multirow{4}{*}{$\begin{array}{l}\text { C18FA- } \\
\text { Zn }\end{array}$} & 0.8 & 0.23 & 1.8 & 42.7 & 852 & 0.20 & 8.9 & \multirow{4}{*}{1.11} & 132.4 & 183.2 & \multirow{4}{*}{5.5} & \multirow{4}{*}{2.3} \\
\hline & 1.3 & 0.30 & 2.5 & 42.7 & 1381 & 0.18 & 6.2 & & 132.7 & 188.1 & & \\
\hline & 1.6 & 0.39 & 3.7 & 42.7 & 1528 & 0.16 & 5.6 & & 132.6 & 190.1 & & \\
\hline & 1.9 & 0.36 & 4.0 & 42.7 & 1619 & 0.15 & 5.0 & & 132.4 & 191.5 & & \\
\hline \multirow{4}{*}{$\begin{array}{c}\text { C16/C18 } \\
\text { FA-Zn }\end{array}$} & 0.7 & 0.19 & 1.3 & 42.1 & 801 & 0.27 & 14.6 & \multirow{4}{*}{1.10} & 125.6 & 146.5 & \multirow{4}{*}{5.3} & \multirow{4}{*}{7.3} \\
\hline & 1.0 & 0.30 & 2.3 & 42.2 & 798 & 0.30 & 12.5 & & 125.4 & 151.1 & & \\
\hline & 1.3 & 0.30 & 3.3 & 42.3 & 885 & 0.28 & 11.9 & & 125.2 & 153.8 & & \\
\hline & 1.9 & 0.28 & 4.1 & 42.4 & 953 & 0.32 & 10.6 & & 125.2 & 158.9 & & \\
\hline $\begin{array}{l}\text { Common } \\
\text { zinc- } \\
\text { stearate }\end{array}$ & 4.9 & 0.54 & 29.5 & 41.8 & 1322 & 0.26 & 12.6 & 1.10 & 124.5 & 164.0 & & \\
\hline
\end{tabular}

*Particle size at $90 \%(\mu \mathrm{m})$ : Particle size when frequencies are counted to $90 \%$.

ing to each peak, and the Miller index (00L) for each diffraction peak on the small-side angle was deduced. The most intensive peak that was considered most suitable for analysis was found to have a Miller index of (003). Therefore, in the present study, we used the (003) to determine the peak intensity and half-band width that indicate the magnitude of crystallinity, and further, we deduced the long spacing that indicates the distance between unit cells, and examined the regularity of molecules in the lateral direction in the fine metallic soap particle, as well as the laminar state of molecular membranes in the longitudinal direction.

The X-ray diffraction patterns of C18FA-Zn synthesized by jet mixing (average diameter $0.8,1.9$, and 3.3 $\mu \mathrm{m})$ and $\mathrm{C} 16 / \mathrm{C} 18 \mathrm{FA}-\mathrm{Zn}$ synthesized by jet mixing

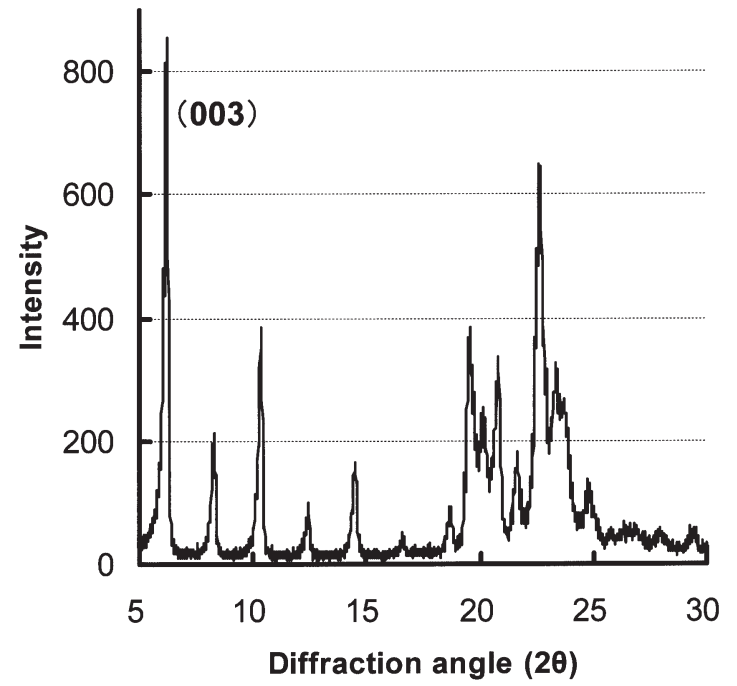

Fig. 5 X-Ray Diffraction Pattern of the C18FA-Zn. The average particle size of C18FA-Zn is $0.8 \mu \mathrm{m}$. 
(average diameter $0.8 \mu \mathrm{m}$ ) in the vicinity of the (003) are shown in Fig. 6. The pattern of C18FA-Zn shows that the diffraction intensity of the (003) peak increases with the increase of the average diameter, and the peak top shifts toward the large angle diffraction side. Comparison of C18FA-Zn and C16/C18FA-Zn, even though they have the same average sizes, the peak top of $\mathrm{C} 16 / \mathrm{C} 18 \mathrm{FA}-\mathrm{Zn}$ is more on the side of the large angle diffraction as compared to that of C18FA-Zn, and the peak is broader. These results suggest that in metallic soap particles, particle size and fatty acid composition influence the crystalline state in the longitudinal and lateral directions.

Table 1 shows the long spacings of the (003) peaks, diffraction intensities, and half-band widths determined in the X-ray diffraction measurements of C14FA-Zn, C16FA-Zn, C18FA-Zn and C16/C18FA-Zn (palmitic acid : stearic acid $=30 \mathrm{wt} \%: 70 \mathrm{wt} \%$ ) that were synthesized by the jet-mixing method, as well as of the common zinc stearate particles produced by pulverizing. Figure 7 shows the relationship of average particle sizes to diffraction intensities and half-band widths in C18FA-Zn, C16FA-Zn and C16/C18FA-Zn (palmitic acid : stearic acid $=30 \mathrm{wt} \%: 70 \mathrm{wt} \%$ ). These results show that in the cases of C16FA-Zn, C18FA-Zn and $\mathrm{C} 16 / \mathrm{C} 18 \mathrm{FA}-\mathrm{Zn}$, the increase of average particle size is associated with an increase of diffraction intensity. Furthermore, a comparison of fine particles of fatty acid zinc salt of the same diameter reveals that the diffraction intensity increases in the order of C14FA-Zn $>$ C16FA-Zn $>$ C18FA-Zn $>$ C16/C18FA-Zn. This indicates that the C16/C18FA-Zn composed of mixed fatty acids has a lower diffraction intensity than C14FA-Zn, C16FA-Zn, and C18FA-Zn, which are composed of a single fatty acid. The half-band width of C18FA-Zn and C16FA-Zn composed of single fatty acids each decreased with the increase of particle size, whereas the half-band width of C16/C18FA-Zn, which is made of mixed fatty acids, tends to increase, though slightly, with the increase of particle size. Comparison of fine particles of fatty acid zinc salt of the same diameter with regard to the half-band width showed that the halfband width increases in the order of C16/C18FA-Zn > C14FA-Zn $>$ C16FA-Zn $>$ C18FA-Zn.

The above results of analysis concerning diffraction intensity and half-band width show that in fine particles of fatty acid zinc salt, whether composed of a single fatty acid or of a mixture of fatty acids, the molecular

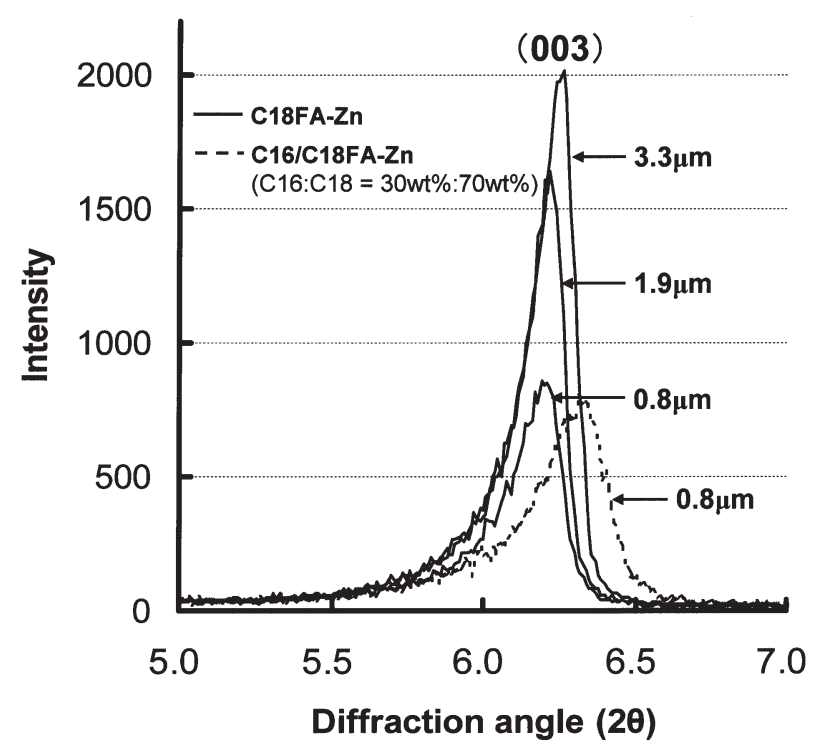

Fig. 6 X-Ray Diffraction Patterns of C18FA-Zn and C16/C18FA-Zn. Average particle sizes of C18FA-Zn are $0.8 \mu \mathrm{m}, 1.9 \mu \mathrm{m}, 3.3 \mu \mathrm{m}$, and of $\mathrm{C} 16 / \mathrm{C} 18 \mathrm{FA}-\mathrm{Zn}$ is $0.8 \mu \mathrm{m}$. The ratio of stearic acid in $\mathrm{C} 16 / \mathrm{C} 18 \mathrm{FA}-\mathrm{Zn}$ is $70 \mathrm{wt} \%$.

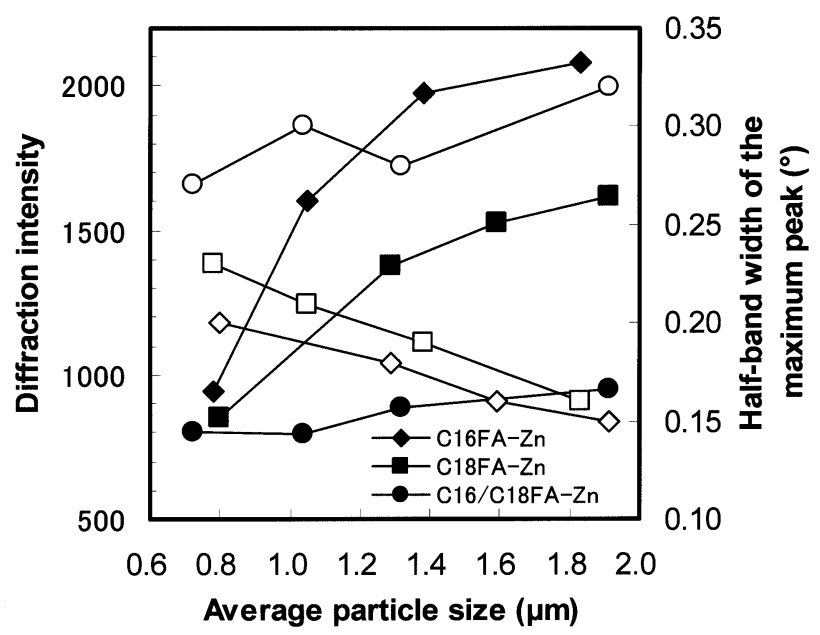

Fig. 7 Relationship between Diffraction Intensities, Half-Band Widths of the Maximum Peaks and Average Particle Sizes of C16FA-Zn, C18FA$\mathrm{Zn}$, and C16/C18FA-Zn. Ratio of stearic acid in C16/C18FA-Zn are $70 \mathrm{wt} \%$. Black-dots are diffraction intensities and white-dots are halfband widths of the maximum peaks. 


\section{K. Sawada and M. Konaka}

regularity in the lateral direction increases with increasing particle size. This phenomenon is considered to be attributable to an increase in the number of molecular arrays in the lateral direction that accompanies the growth of fatty acid zinc salt particles. The fine particles of fatty acid zinc salt composed of a single fatty acid show an increase of molecular regularity in the lateral direction also with the increase of the length of fatty acid chain. This is considered to be attributable to an increase of intermolecular force that occurs when the fatty acid chains become longer. C16/C18FA-Zn composed of a mixture of fatty acids, on the other hand, tends to have a relatively low crystallinity as compared to those consisting of single fatty acids. This is considered to be due to lattice strain, such as heterogeneous strain, which results from the existence of diverse fatty chains. Crystallinity of fine metallic soap particles composed of a mixture of fatty acids will be discussed in detail later.

\section{3·3 Relationship between Particle Size, Density and Heat Characteristics of Fine Particles of Fatty Acid Zinc Salt}

True density $(\rho)$, melting point and absorbed heat $(\Delta \mathrm{H})$ of C14FA-Zn, C16FA-Zn, C18FA-Zn, and C16/C18FA-Zn (palmitic acid: stearic acid $=30 \mathrm{wt} \%$ : $70 \mathrm{wt} \%$ ) produced by jet mixing and those of the common zinc stearate produced by pulverization are shown in Table 1. It shows that the true density of C14FA-Zn, C16FA-Zn and C18FA-Zn decreases with the increase of the length of fatty acid chains in the fatty acid zinc salt. The weight of zinc in $1 \mathrm{~cm}^{3}$ of C14FA-Zn, C16FA-Zn and C18FA-Zn, determined on the basis of true density, was $0.146 \mathrm{~g}, 0.129 \mathrm{~g}$, and $0.116 \mathrm{~g}$, respectively, showing that the zinc weight decreased with increasing of the length of fatty acid chain. The weight of the remaining component (fatty acid chain), on the other hand, was almost the same for all the fatty acid zinc salts (ranging from $1.00 \mathrm{~g}$ to $1.01 \mathrm{~g}$ ), suggesting that the decrease of true density accompanying the growth of the length of fatty acid chain is due to the decreased density of the metal. For C16/C18FA-Zn composed of a mixture of fatty acids, the true density can be estimated to be 1.12 based on the composition ratio of the fatty acids; however, the actual measurement shows a smaller value of 1.10. Based on actual measurements, the weight of zinc present in $1 \mathrm{~cm}^{3}$ of $\mathrm{C} 16 / \mathrm{C} 18 \mathrm{FA}-\mathrm{Zn}$ is calculated to be $1.17 \mathrm{~g}$, which is about the same as the weight of zinc in $1 \mathrm{~cm}^{3}$ of C18FA-Zn. The weight of the remaining component (fatty chain portion) is $0.98 \mathrm{~g}$, which is less than that of C18FA-Zn. These results suggest that the fatty acid zinc salt consisting of a mixture of fatty acids contains voids in the solid state. In 3.2, we attributed the decreased crystallinity of fatty acid zinc salt consisting of a mixture of fatty acids to the development of lattice strain, such as heterogeneous strain, resulting from the existence of diverse fatty chains. The voids within the metallic soap are considered to correspond to the gaps that develop in the lattice under strain. The above results suggest that the crystallinity of fatty acid zinc salt composed of a mixture of fatty acids is lower because the intermolecular force in the longitudinal direction is reduced due to development of voids and because the intermolecular force in the lateral direction between the fatty acid chains is reduced as a result of randomization of fatty acid chains.

From the values of melting point and $\Delta \mathrm{H}$ in Table 1, we notice that in all fine particles of fatty acid zinc salt, $\Delta \mathrm{H}$ rises with the increase of particle size. We reported in $\mathbf{3} \cdot \mathbf{2}$ that the molecular regularity in the lateral direction increases with increasing particle size, thus resulting in a rise of crystallinity. The above result is considered to be due to the increased intermolecular force resulting from the enhanced molecular regularity, which means that the melting of a metallic soap having enhanced molecular regularity requires more energy, thus resulting in a rise of $\Delta \mathrm{H}$. Comparison of C14FA$\mathrm{Zn}, \mathrm{C16FA}-\mathrm{Zn}, \mathrm{C18FA}-\mathrm{Zn}$, all consisting of a single fatty acid, with $\mathrm{C} 16 / \mathrm{C} 18 \mathrm{FA}-\mathrm{Zn}$ consisting of a mixture of fatty acids with regard to melting point and $\Delta \mathrm{H}$ at same particle sizes, revealed that the fatty acid zinc salt composed of a mixture of fatty acids has a lower melting point and $\Delta \mathrm{H}$ than fatty acid zinc salts composed of a single fatty acid. Fine particles of fatty acid zinc salt composed of a mixture of fatty acids are suggested to have a reduced intermolecular force in the lateral and longitudinal directions due to voids existing between lattices, and this is considered to cause a lower melting point and $\Delta \mathrm{H}$.

\subsection{Relationship between Specific Surface Area and Particle Size of Fine Fatty Acid Zinc Salts}

Results of specific surface area measurements concerning C14FA-Zn, C16FA-Zn, C18FA-Zn, and 
C16/C18FA-Zn (palmitic acid : stearic acid $=30 \mathrm{wt} \%$ : $70 \mathrm{wt} \%$ ) produced by jet mixing and the common zinc stearate produced by pulverization are shown in Table 1. With some additional data, the relationship between average particle sizes and specific surface areas are shown in Fig. 8 for C16FA-Zn, C18FA-Zn, and C16/C18FA-Zn. These results show that in any of the fine particles of fatty acid zinc salt, there is a decrease of specific surface area when the particle size is increased. While C16FA-Zn and C18FA-Zn, both of which are composed of a single fatty acid, have similar specific surface areas, C16/C18FA-Zn, composed of a mixture of fatty acids, was found to have a larger specific surface area than C16FA-Zn and C18FA-Zn of the same particle size. The specific surface area of zinc stearate obtained by the common pulverizing method is similar to that of $\mathrm{C} 16 / \mathrm{C} 18 \mathrm{FA}-\mathrm{Zn}$ with a particle size of $1.0 \mu \mathrm{m}$. This is probably due to the increase of surface area resulting from the many fracture surfaces created during the pulverization process.

Gas sorption was used to determine the specific surface area. In this method, the surface of the particle is allowed to adsorb gas molecules with a known area covered by each adsorbed molecule, and from the amount of the adsorbed gas, the specific surface area of the sample can be determined (13). If voids are assumed to be present within the metallic soap as described in $\mathbf{3} \cdot \mathbf{3}$, the value of the specific surface area will be the sum of the surface area of the particle plus the surface area of the voids within the particle. Particularly, lamellar-shaped particles, as is the case with the fine metallic soap, are expected to allow accurate measurement of intra-particle voids because they have large exposed surface areas. Therefore, we considered it possible to create a function for the relationship between the particle size and specific surface area of each fine particle of fatty acid zinc salt with two parameters, i.e., (1) parameter of particle surface area that changes with the change in particle size, and (2) gas adsorbing surface area, excluding the particle surface area (which corresponds to the amount of voids present within the particle). We tried to quantify the amount of voids existing within the particles based on the relationship between the measured specific surface area and particle size. For the particle surface area parameter (1), we made the assumption that it was formed from the theoretical surface area value that can be calculated from an ideal molecular shape and a correction factor. For

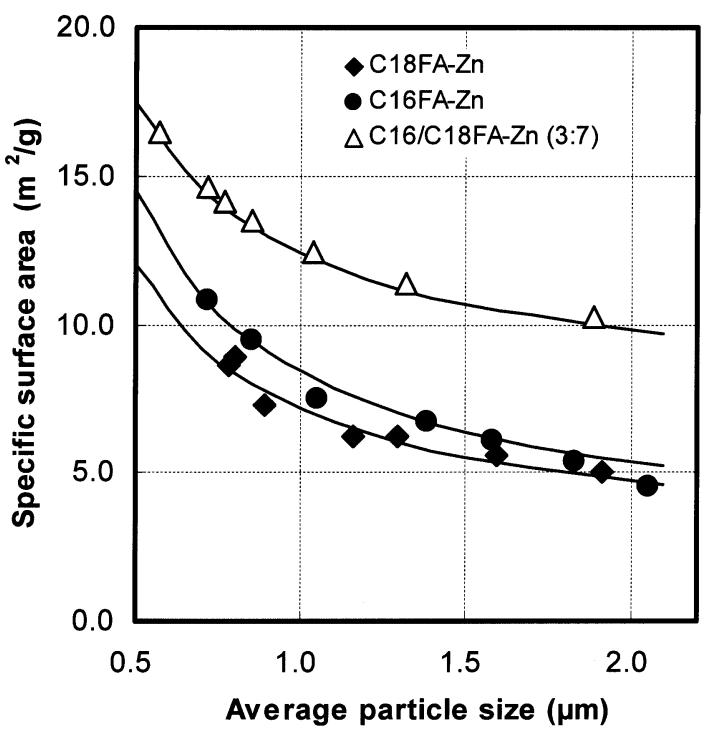

Fig. 8 Relationship between Specific Surface Areas and Average Particle Sizes of C16FA-Zn, C18FA-Zn, and C16/C18FA-Zn. Ratio of stearic acid in C16/ C18FA-Zn are $70 \mathrm{wt} \%$.

example, the shape of fine particles of fatty acid zinc salt is assumed to be a cylinder with its thickness being $1 / 10$ of its diameter. Taking the average particle size as the representing value of its diameter, the surface area per unit volume $A\left(\mathrm{~m}^{2} / \mathrm{g}\right)$ of fine particles of fatty acid zinc salt with an average particle size $\mathrm{r}(\mu \mathrm{m})$ and true density $\rho\left(\mathrm{g} / \mathrm{cm}^{3}\right)$ can be calculated by the following Equation 2. $\mathrm{X}$ and $\mathrm{y}$ in the equation represent the correction factor of the theoretical surface area value and the amount of voids existing within the particle, respectively.

$$
A=\frac{24 X}{r \rho}+y
$$

The correction factor (x) and the amount of voids present within the particle (y) used in Equation 2 were fitted to each of the measured values of specific surface areas shown in Fig. 8. The values of $x$ and $y$ for each fine particle of fatty acid zinc salt are shown in Table $\mathbf{1 .}$ The simulation results of the specific surface area obtained on the basis of these values for each fine particle of fatty acid zinc salt are shown in Fig. 8 in full lines. The simulation lines obtained from Equation 2 are found to be in good agreement with the lines of actual measurements. The values of $\mathrm{x}$ in Table 1 range between 0.24 and 0.29 for C16FA-Zn, C18FA-Zn and 


\section{K. Sawada and M. Konaka}

C16/C18FA-Zn, regardless of the length of the respective fat chain or whether only a single fatty acid or a mixture of fatty acids is involved. This suggests that the factors related to the particle properties, such as particle size distribution and particle shape, are similar for all fine particles of fatty acid zinc salt, regardless of the length of the fatty acid chain or whether only a single fatty acid or a mixture of fatty acids is involved. In contrast, the y values in Table 1 for C16FA-Zn and C18FA-Zn, which are composed of a single fatty acid, are $2.3 \mathrm{~m}^{2} / \mathrm{g}$, while for $\mathrm{C} 16 / \mathrm{C} 18 \mathrm{FA}-\mathrm{Zn}$ it is as large as $7.3 \mathrm{~m}^{2} / \mathrm{g}$. There seem to be $5.0 \mathrm{~m}^{2} / \mathrm{g}$ more voids in the C16/C18FA-Zn particles than in the C16FA-Zn or C18FA-Zn particles, which are composed of a single fatty acid. So far, the presence of voids between fat chains in mixed fatty acids has been predicted (9), but it was not possible to elucidate their concrete amount. Under these circumstances, the attempt to calculate the concrete amount of voids in fatty acid chains is considered very productive.

\subsection{Relationship Between Crystal Struc- ture, Heat Characteristics and Fatty Acid Composition of Fine Fatty Acid Zinc Salts}

Using the jet-mixing method, we synthesized C16/C18FA-Zn particles containing stearic acid in the concentration range from $0 \%$ to $100 \%$ by weight and conducted X-ray diffraction measurements of the particles. In the same way as in $\mathbf{3} \cdot \mathbf{2}$, long spacing, diffraction intensity, and half-band width in the (003) were determined and their relation to fatty acid composition was examined. The results are shown in Table 2. To reduce the influence of particle size on X-ray diffraction as explained in $\mathbf{3} \cdot \mathbf{2}$, the average particle sizes were set at about $2 \mu \mathrm{m}$. The particles were also similar with regard to the values of standard deviation and particle size at $90 \%$. Figure 9 shows the X-ray diffraction patterns in the vicinity of the (003) when the ratio of stearic acid in fatty acid composition was changed from $0 \%$ to $100 \%$. The results show that the $\mathrm{C} 16 / \mathrm{C} 18 \mathrm{FA}-\mathrm{Zn}$ composed of a mixture of fatty acids has one single (003) peak. This suggests that within C16/C18FA-Zn, same fatty acid chains are not next to each other, but are aligned randomly. In comparison to C16FA-Zn and C18FA-Zn, which consist of a single fatty acid, C16/C18FA-Zn particles have relatively broad peaks, whose shape and intensity change in various ways according to the change in fatty acid composition.

Table 2 shows the values of diffraction intensity and half-band width obtained from the analysis of the X-ray diffraction patterns of Fig. 9. The relationships of fatty acid composition to diffraction intensities and half-band widths are shown in Fig. 10. It shows that C16/C18FA$\mathrm{Zn}$ consisting of a mixture of fatty acids has a lower diffraction intensity and larger half-band width than C16FA-Zn and C18FA-Zn, which are composed of a single fatty acid. These results are considered to be attributable to the decreased crystallinity in the longitudinal and lateral directions resulting from random molecular alignment in the lateral direction. What seems even more interesting is that this decrease of crystallinity changes with the mixture ratio of fatty acids. The crystallization rate increases when the composition ratio of stearic acid and palmitic acid is about 50:50 by weight. This phenomenon suggests that, in line with the change in fatty acid composition, the fatty acid alignment structure changes from rough to dense and again to rough. Especially when the ratio of stearic acid to palmitic acids becomes 50:50, the molecules seem to be aligned more densely in the longitudinal and lateral directions.

The relationship of fatty acid compositions to melting points and values of $\Delta \mathrm{H}$ is shown in Table 2 and Fig. 11. Melting points and $\Delta \mathrm{H}$ were found to change in line with the change in fatty acid composition. C16/C18FA-Zn consisting of a mixture of fatty acids tend to have a lower melting point and $\Delta \mathrm{H}$ than C16FA$\mathrm{Zn}$ and C18FA-Zn, both consisting of a single fatty acid. There is also a relationship between the changes of crystallinity shown in Fig. $\mathbf{1 0}$ and melting points and $\Delta \mathrm{H}$; the fatty acid composition presenting higher crystallinity was associated with higher melting point and $\Delta \mathrm{H}$. Similarly to the explanation in $\mathbf{3} \cdot \mathbf{3}$, this tendency is explained by the influence of the change of intermolecular force resulting from random molecular alignment and the presence of voids between lattices.

The values of long spacing derived from the analysis of the X-ray diffraction patterns in Fig. 9 are shown in Table 2. The relationship between fatty acid compositions and long spacings are shown in Fig. 12. Long spacings change in line with the mixture ratio of fatty acids, and the long spacing of C16FA-Zn was found to increase greatly by addition of stearic acid. For example, in case the ratio of palmitic acid to stearic acid is $80 \%$ to $20 \%$ by weight, the long spacing is 39.8 , 
Table 2 Results of Particle Size Distribution Analysis, X-Ray Diffraction Analysis and Differential Scanning Calorimetry Analysis for Fine Metallic Soap Particles (C16/C18FA-Zn). Ratios of stearic acid in C16/C18FA-Zn are 0 wt $\%, 20$ wt $\%, 40 \mathrm{wt} \%, 50 \mathrm{wt} \%, 60 \mathrm{wt} \%, 70 \mathrm{wt} \%, 80 \mathrm{wt} \%$ and $100 \mathrm{wt} \%$.

\begin{tabular}{|c|c|c|c|c|c|c|c|c|}
\hline $\begin{array}{c}\text { Ratio of C18-FA } \\
\text { (Weight } \% \text { ) }\end{array}$ & $\begin{array}{c}\text { Average } \\
\text { particle size } \\
(\mu \mathrm{m})\end{array}$ & $\begin{array}{l}\text { Standard } \\
\text { deviation }\end{array}$ & $\begin{array}{c}\text { Particle size } \\
\text { at } 90 \% * \\
(\mu \mathrm{m})\end{array}$ & $\begin{array}{c}\text { Long } \\
\text { spacing }\end{array}$ & $\begin{array}{l}\text { Diffraction } \\
\text { intensity }\end{array}$ & $\begin{array}{l}\text { Half-band width } \\
\text { of the maximum } \\
\text { peak }\end{array}$ & $\begin{array}{c}\text { Melting } \\
\text { point } \\
\left({ }^{\circ} \mathrm{C}\right)\end{array}$ & $\begin{array}{c}\Delta \mathrm{H} \\
(\mathrm{mJ} / \mathrm{mg})\end{array}$ \\
\hline 100 & 1.9 & 0.36 & 4.0 & 42.7 & 1619 & 0.15 & 132.4 & 186 \\
\hline 80 & 2.0 & 0.31 & 4.8 & 42.7 & 1206 & 0.38 & 126.3 & 139 \\
\hline 70 & 1.9 & 0.28 & 4.1 & 42.4 & 953 & 0.32 & 125.8 & 129 \\
\hline 60 & 1.9 & 0.27 & 4.0 & 41.2 & 1348 & 0.28 & 127.1 & 151 \\
\hline 50 & 2.1 & 0.22 & 3.9 & 40.6 & 1619 & 0.21 & 127.9 & 176 \\
\hline 40 & 2.0 & 0.25 & 4.2 & 40.4 & 1107 & 0.32 & 125.5 & 168 \\
\hline 30 & 2.1 & 0.37 & 5.7 & 40.2 & 1128 & 0.37 & 125.8 & 169 \\
\hline 20 & 2.1 & 0.31 & 5.0 & 39.8 & 1411 & 0.27 & 127.9 & 175 \\
\hline 0 & 2.1 & 0.26 & 4.3 & 38.3 & 2138 & 0.20 & 132.2 & 189 \\
\hline
\end{tabular}

*Particle size at $90 \%(\mu \mathrm{m})$ : Particle size when frequencies are counted to $90 \%$.

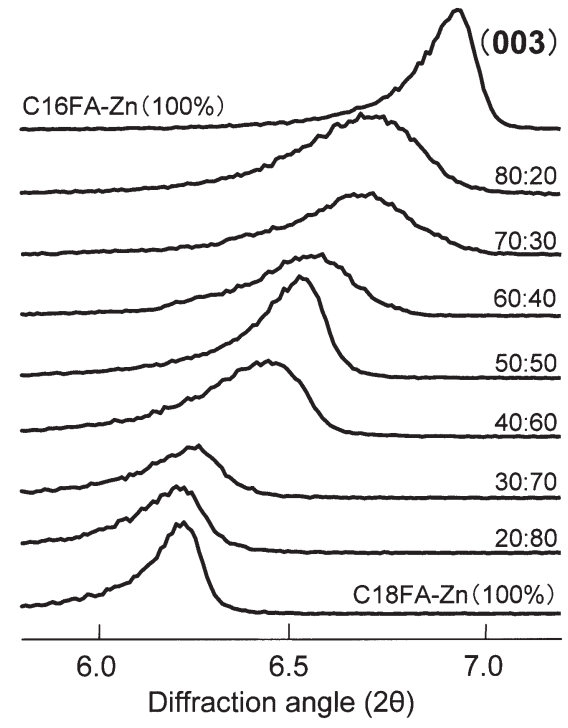

Fig. 9 X-Ray Diffraction Patterns of C16/C18FAZn. Ratios of stearic acid in C16/C18FA-Zn are $0 \mathrm{wt} \% \sim 100 \mathrm{wt} \%$, and average particle sizes for samples are $2 \mu \mathrm{m} \pm 0.1 \mu \mathrm{m}$.

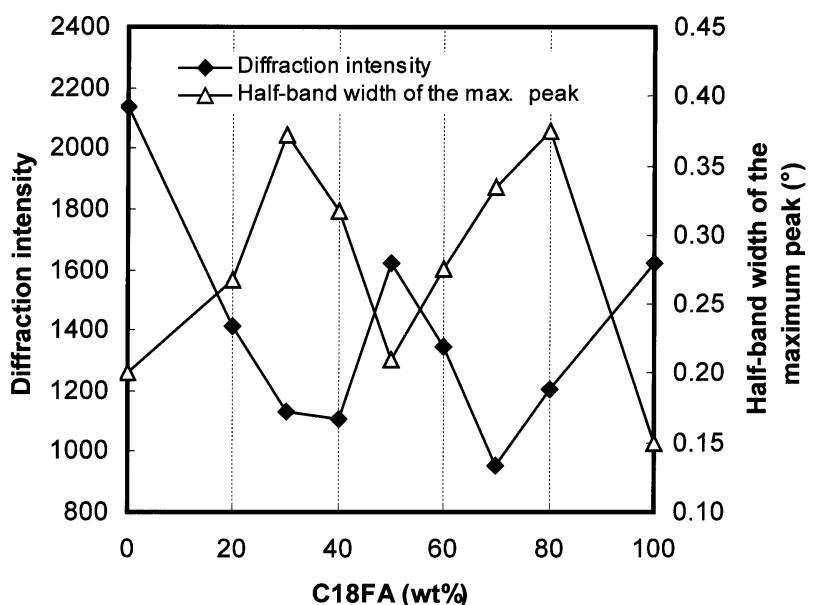

Fig. 10 Relationship between Diffraction Intensities, Half-Band Widths of the Maximum Peaks and Ratios of Stearic Acid in C16/C18FA-Zn. Ratios of stearic acid in C16/C18FA-Zn are $0 \mathrm{wt} \% \sim 100$ $\mathrm{wt} \%$, and average particle sizes for these samples are $2 \mu \mathrm{m} \pm 0.1 \mu \mathrm{m}$. 


\section{K. Sawada and M. Konaka}

which is a value approximately quite close to that (40.6) when the ratio is 50:50, i.e., palmitic acid and stearic acid are contained at a molar ratio of approximately $1: 1$. Increasing the ratio of stearic acid even further from 50:50, there is again a substantial increase of long spacing of C16/C18FA-Zn. For example, at a palmitic acid to stearic acid ratio of $30: 70$ by weight, the long spacing is 42.2 , which is close to that (42.7) of C18FA$\mathrm{Zn}$. The long spacing of fatty acid zinc salt consisting of a mixture of fatty acids was found to have two inflection points in relation to the change of fatty acid composition.

\subsection{Representation of Crystalline Struc- ture of Fine Metallic Soap Particles}

In $\mathbf{3} \cdot \mathbf{4}$, we demonstrated that there are certain amounts of voids between molecules in fatty acid zinc salt composed of a mixture of fatty acids. However, regarding the site where the voids develop, the discussions in $\mathbf{3 \cdot 4}$ are not sufficient. Long spacings correspond to the distances between unit cells in the longitudinal direction in the crystalline structure. Therefore, a change in long spacing in line with the change of fatty acid composition suggests that the distances between the layers are changing. Based on these results, changes of the crystalline state associated with the

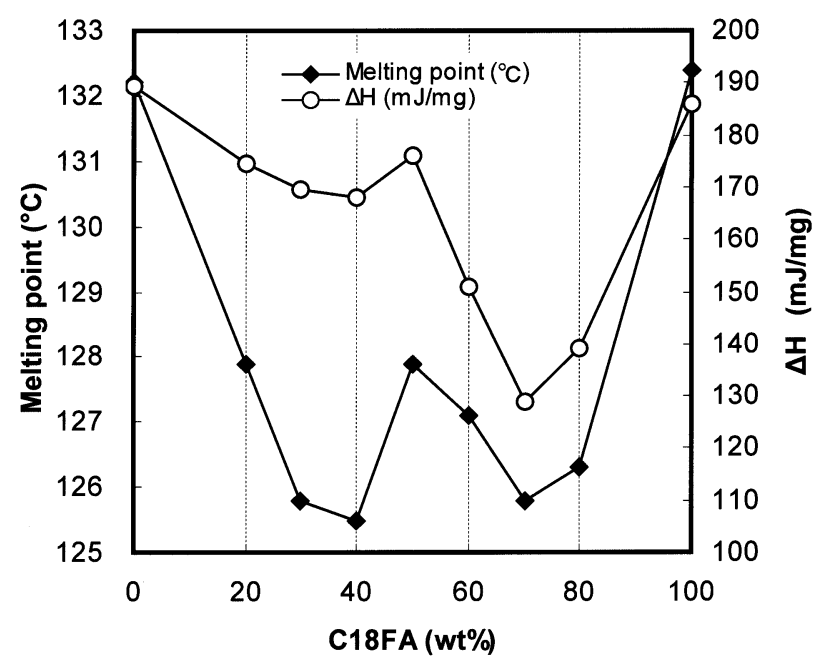

Fig. 11 Relationship between Melting Points, Values of $\Delta \mathrm{H}$ and Ratios of Stearic Acid in C16/C18FA-Zn. These $\Delta \mathrm{H}$ were measured by DSC. Ratios of stearic acid in C16/C18FA-Zn are $0 \mathrm{wt} \% \sim 100$ $\mathrm{wt} \%$, and average particle sizes for these samples are $2 \mu \mathrm{m} \pm 0.1 \mu \mathrm{m}$. changes in fatty acid composition are summarized in Fig. 13. The crystal structures corresponding to each fatty acid composition are explained in the following:

1) The crystal structures of C16FA-Zn and C18FA-Zn composed of a single fatty acid correspond to the structure models on both sides of the diagram in Fig. 12. The molecules are thought to be densely aligned, both in the longitudinal and lateral directions, in these fatty acid zinc salts.

2) In C16/C18FA-Zn particles composed of palmitic acid and stearic acid at a weight ratio of 50:50, palmitic acid and stearic acids are thought to be aligned almost alternately, as shown in the middle of the diagram in Fig. 12. In such an alignment, the stearic acid molecules supplement the lack of fat chain length of the palmitic acid molecules, resulting in a dense alignment of molecules both in the longitudinal and lateral directions and a great degree of crystallization of C16/C18FA-Zn. C16/C18FA-Zn with this composition ratio shows a higher melting point and higher energy absorbed during melting. The long spacing is thought to be equal to the median of the long spacings of zinc palmitate and zinc stearate, and it is in good agreement with the long spacing value shown in Fig. 11.

3) The crystal structure of a C16/C18FA-Zn that con-

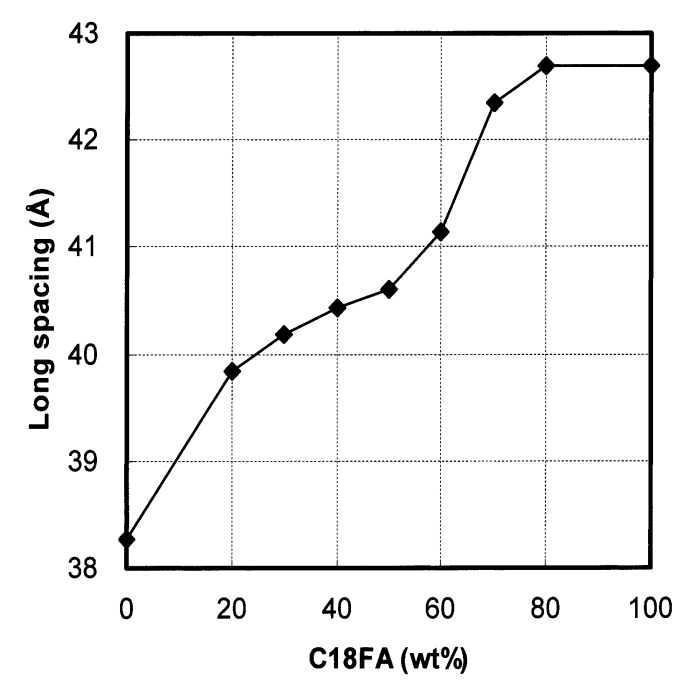

Fig. 12 Relationship between Long Spacings and Ratios of Stearic acid in C16/C18FA-Zn. Ratios of stearic acid in C16/C18FA-Zn are $0 \mathrm{wt} \% \sim 100$ $\mathrm{wt} \%$, and average particle sizes for these samples are $2 \mu \mathrm{m} \pm 0.1 \mu \mathrm{m}$. 
tains palmitic acid at a higher ratio is influenced by the distance between molecular crystal membranes of palmitic acid and stearic acid. Therefore, space is created between two palmitic acid molecules as shown by the dotted lines and causes a reduction of the degree of crystallization. In the C16/C18FA-Zn with such fatty acid composition, melting point and absorbed heat decreased. The long spacing of such particles is expected to be closer to the long spacing of the C16/18FA-Zn with a 50:50 fatty acid composition. It is in good agreement with the value of long spacing in Fig. 11.

4) In the case of the $\mathrm{C} 16 / \mathrm{C} 18 \mathrm{FA}-\mathrm{Zn}$ that contains stearic acid at a higher ratio, the crystal structure is affected by the distance between molecular crystal membranes of stearic acid. The spaces between crystal membranes of palmitic acid molecules or of palmitic acid and stearic acid molecules create more voids, as shown by the dotted lines, resulting in yet lower crystallinity. The melting point and absorbed heat of melting of C16/C18FA-Zn with such composition are lower, and its long spacing is considered to be closer to that of C18FA-Zn. This is in good agreement with the long spacing value in Fig. 11.

These results indicate that the diagram in Fig. 12 may well explain the X-ray diffraction results, i.e. the changes in the long spacing and crystallinity that accompany the change in fatty acid composition. To date, several analyses have been performed on the crystal structure of fatty acid derivatives as represented by metallic soaps (8-10). However, no detailed discussion of the relation between fatty acid composition and the crystalline state was possible because it was difficult to unify processing conditions and obtain solid particles of homogenous shape. Under these circumstances, the present study to attempt to elucidate the relation between fat chain alignment and fatty acid composition using fine metallic soap particles is considered to be extremely productive.

\section{Conclusion}

The development of a new process called jet mixing made it possible to synthesize and isolate fine metallic soap particles with new properties not available with common metallic soaps. Fine particles synthesized by jet mixing are by far more minute than common metallic soap particles, and have narrower particle size distribution and the characteristic shape of thin layers. Jet mixing also enables the arbitrary control of particle sizes without pulverizing. Using the particles synthesized by jet mixing, we studied the relationship of the particle size to crystalline state, heat characteristics, and specific surface area in fatty acid zinc salt as a representative metallic soap. The results of our study revealed that the crystalline state, melting point, amount of heat of melting, and specific surface area of fatty acid zinc salt particles change in line with the changes in particle size and that the crystallinity rises with the increase of particle size. The rise in crystallinity is associated with a rise in the melting point and amount of heat absorption during melting. Based on the results of specific surface area analysis, we were able to estimate the

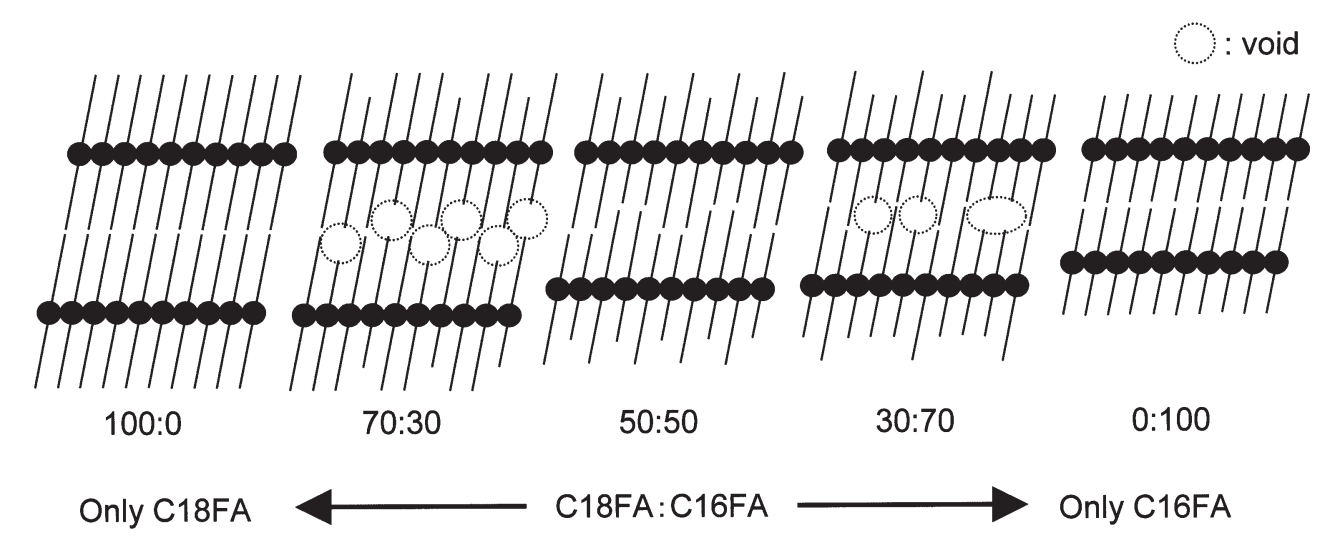

Fig. 13 Crystal Structure Models of C16/C18FA-Zn. Weight ratios of Stearic acid in these models are $0 \%, 30 \%, 50 \%, 70 \%$ and $100 \%$. Dot-line circles mean void areas in these models. 


\section{K. Sawada and M. Konaka}

amount of voids within the fatty acid zinc salt particles composed of a mixture of fatty acids. We further examined the influence of changes in fatty acid composition on crystalline state, melting point and heat absorption during melting and found out that the changes in fatty acid composition cause diverse changes of crystalline state, melting point, and amount of heat absorption during melting of fatty acid zinc salt. It was found out that the long spacing determined by X-ray diffraction analysis has two points of inflexion centering around the long spacing of the fatty acid zinc salt with a composition ratio of 50:50 by weight. On the basis of these results, we illustrated the relationship between crystalline structure and fatty acid composition in a diagram that could explain very well the results of analyses obtained to date. These series of analyses were made possible for the first time through completion of the new production method of jet mixing that allows synthesis of solid particles with homogenous shape under homogenous processing conditions. We expect that by expanding research into the structure of fatty acids that has been difficult to analyze using such particle models, more significant knowledge will be accumulated.

\section{References}

1. T. KAWAI, Everything for Nano-technologies, Kogyo Chosakai Publishing (2001).
2. H. YANAGIDA, Engineering System for Fine Particles -Applied Technology II, Fujitec (2002).

3. T. YOSHIDA, Nature and Application for Metallic Soap, Saiwaishobo (1988).

4. K. SAWADA and M. KONAKA, Preparation of Sub-Micron Particles of Fatty Acid Multivalent Metal Salt Using Newly Developed Jet-Mixing Method, J. Soc. Powder Technol., Vol. 40, 565-572 (2003).

5. K. SAWADA, Turn of Events for Metallic Soaps - Approach to Miniaturization for Metallic Soaps, Preprint of 42th Summer workshop, Oleochem. Soc. Jpn., 9-21 (2002).

6. K. SAWADA, Lubricants-Standard Lubricants and Development for New Lubricants, Plastics Age, Vol. 48, 137-142 (2002).

7. K. SAWADA, New Characteristic Additives designed for Imaging Toner -Fine Metallic Soaps \& High-Purified Solid Esters-, Final Program and Proceedings of NIP19 International Conference on Digital Printing Technologies, New Orleans, Louisiana, IS \& T, 139 (2003).

8. M. GOTO, X-Ray Diffraction and Polymorphism of Triglycerides, Yukagaku., Vol. 19, 583-591 (1970).

9. K. OGUNO and T. SAITO, X-Ray Diffraction Studies of the Metal Soaps of Fatty Acids of Odd Carbon Numbers, Bull. Chem. Soc. Jpn., Vol. 50, 1624 (1977).

10. E. STANLEY, An Interpretation of the Long-Spacing Intensities of Calcium Stearate Monohydrate, Nature, Vol. 4447, 165 (1955).

11. R.D. VOID and G.S. HATTIANGDI, Characterization of Heavy Metal Soaps by X-Ray Diffraction, Ind. Eng. Chem., Vol. 41, 2311 (1949).

12. Wing SALD-2100 Operating Manual, 26, Shimazu Corp.

13. M. ARAKAWA, Schematic Description of Powder's Properties, Soc. Powder Technol. Jpn., 103-117 (1985). 